\title{
Outcomes in transcranial microsurgery versus extended endoscopic endonasal approach for primary resection of adult craniopharyngiomas
}

\author{
*Todd J. Wannemuehler, MD, ${ }^{1}$ Kolin E. Rubel, BS, ${ }^{1}$ Benjamin K. Hendricks, MD, ${ }^{2}$ \\ Jonathan Y. Ting, MD, ${ }^{1}$ Troy D. Payner, MD, ${ }^{2}$ Mitesh V. Shah, MD, ${ }^{2}$ and \\ Aaron A. Cohen-Gadol, MD, MSc ${ }^{2}$ \\ 1Department of Otolaryngology-Head \& Neck Surgery; and ${ }^{2}$ Goodman Campbell Brain and Spine, Department of Neurological \\ Surgery, Indiana University School of Medicine, Indianapolis, Indiana
}

\begin{abstract}
OBJECTIVE Craniopharyngiomas have historically been resected via transcranial microsurgery (TCM). In the last 2 decades, the extended endoscopic endonasal (transtuberculum) approach to these tumors has become more widely accepted, yet there remains controversy over which approach leads to better outcomes. The purpose of this study is to determine whether differences in outcomes were identified between TCM and extended endoscopic endonasal approaches (EEEAs) in adult patients undergoing primary resection of suprasellar craniopharyngiomas at a single institution.

METHODS A retrospective review of all patients who underwent resection of their histopathologically confirmed craniopharyngiomas at the authors' institution between 2005 and 2015 was performed. Pediatric patients, revision cases, and patients with tumors greater than 2 standard deviations above the mean volume were excluded. The patients were divided into 2 groups: those undergoing primary TCM and those undergoing a primary EEEA. Preoperative patient demographics, presenting symptoms, and preoperative tumor volumes were determined. Extent of resection, tumor histological subtype, postoperative complications, and additional outcome data were obtained. Statistical significance between variables was determined utilizing Student t-tests, chi-square tests, and Fisher exact tests when applicable.
\end{abstract}

RESULTS After exclusions, 21 patients satisfied the aforementioned inclusion criteria; 12 underwent TCM for resection while 9 benefitted from the EEEA. There were no significant differences in patient demographics, presenting symptoms, tumor subtype, or preoperative tumor volumes; no tumors had significant lateral or prechiasmatic extension. The extent of resection was similar between these 2 groups, as was the necessity for additional surgery or adjuvant therapy. CSF leakage was encountered only in the EEEA group (2 patients). Importantly, the rate of postoperative visual improvement was significantly higher in the EEEA group than in the TCM group ( $88.9 \%$ vs $25.0 \% ; p=0.0075)$. Postoperative visual deterioration only occurred in the TCM group (3 patients). Recurrence was uncommon, with similar rates between the groups. Other complication rates, overall complication risk, and additional outcome measures were similar between these groups as well.

CONCLUSIONS Based on this study, most outcome variables appear to be similar between TCM and EEEA routes for similarly sized tumors in adults. The multidisciplinary EEEA to craniopharyngioma resection represents a safe and compelling alternative to TCM. The authors' data demonstrate that postoperative visual improvement is statistically more likely in the EEEA despite the increased risk of CSF leakage. These results add to the growing evidence that the EEEA may be considered the approach of choice for resection of select confined primary craniopharyngiomas without significant lateral extension in centers with experienced surgeons. Further prospective, multiinstitutional collaboration is needed to power studies capable of fully evaluating indications and appropriate approaches for craniopharyngiomas. https://thejns.org/doi/abs/10.3171/2016.9.FOCUS16314

KEY WORDS craniotomy; endoscopic; transsphenoidal; craniopharyngioma; suprasellar; complications; outcomes; adult

ABBREVIATIONS DI = diabetes insipidus; EEEA = extended endoscopic endonasal approach; $E O R=$ extent of resection; $T C M=$ transcranial microsurgery. SUBMITTED July 31, 2016. ACCEPTED September 12, 2016.

* Mr. Rubel and Dr. Hendricks contributed equally to this work. 


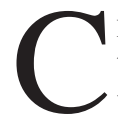
RANIOPHARYNGIOMAS are midline suprasellar brain tumors that often extend superiorly into the third ventricle and are composed of benign epithelial tissue. ${ }^{13,16,25}$ These tumors are thought to derive embryologically from remnant cell rests after failure of the craniopharyngeal duct of Rathke's pouch to entirely disappear during development. ${ }^{24,25}$ Other midline suprasellar tumors that may also occupy this location include meningiomas, pituitary adenomas, gliomas, and germinomas, among others. ${ }^{15}$ The incidence of craniopharyngioma is estimated at approximately 1 to 3 per 100,000 and occurs without sex or racial predilection. ${ }^{1,13}$ Diagnosis and referral to neurological surgery is often delayed because of the tumor's slow growth and the insidious onset of symptoms, including headache, endocrine dysfunction, and visual disturbance, classically in the form of bitemporal hemianopia.1,10,13 Gross-total resection of these tumors represents the gold standard of treatment, although subtotal resection with adjuvant radiotherapy remains an alternative option when the tumor cannot be completely excised without placing the critical cerebrovascular structures at risk. ${ }^{13,16}$ The quality of life after resection is an important priority.

Craniopharyngiomas occupy a critical central anatomical location close to the third ventricle, the optic chiasm and tracts, the internal carotid arteries and their branches, and the pituitary stalk. ${ }^{13}$ Historically, neurosurgeons have employed a variety of transcranial microsurgery (TCM) corridors for reaching and removing these tumors, including the anterolateral pterional and orbitozygomatic, anterior subfrontal and bifrontal, and transcallosal-transventricular approaches. ${ }^{29,32,33}$ All of these TCM approaches share the risk of brain retraction and, importantly, place the optic nerve between the surgeon and the tumor. The optic nerves must be manipulated for exposure of the tumor, and the superior pole of the mass is placed within the operative blind spot. These factors increase the risk of postoperative neurological deficits, including visual deterioration. ${ }^{17,21,33}$ Other complications include endocrinopathy and stroke..$^{910,17,22}$

Over the last decade, refinements in extended endoscopic endonasal approaches (EEEAs) have encouraged more surgeons to use this route. , $3,23,27$ EEEA resection of craniopharyngiomas employs expanded transsphenoidal craniocaudal access via transtuberculum, transplanum, and transclival osteotomies. ${ }^{2,15,18}$ EEEA is a sensible approach for a subchiasmatic lesion extending into the third ventricle as it allows for exploration via a subchiasmatic operative corridor. Importantly, the tumor is debulked, and the optic apparatus is decompressed early before the nerves are handled. In addition, the tumor is exposed along its long axis; therefore, extended operative working angles are created for maximizing tumor resection while minimizing surgical blind spots. Finally, brain retraction and manipulation is avoided. ${ }^{4-6,12,17}$ The increased rate of CSF leakage and the limited selection of tumors that are appropriate candidates for this approach are some of the drawbacks of this corridor. ${ }^{12,15,16}$ Tumors with lateral extension beyond the carotid bifurcation or with vascular encasement are considered poor candidates for EEEA. In addition, the required specialized expertise limits the use of the approach. To date, no study has specified the pre- cise number of cases or training requirements to obtain the necessary experience to be successful in using these endoscopic approaches.

There is great heterogeneity in the operative care of craniopharyngiomas across different institutions, with limited evidence regarding the comparative benefits of TCM and EEEA routes. In 2012, Komotar et al. performed a meta-analysis of 88 retrospective case series to compare outcomes of TCM and EEEA for craniopharyngiomas. ${ }^{17}$ Since that time, only one single-institution series has compared the outcomes of TCM and EEEA in the hands of a single group of surgeons. ${ }^{15}$ The studies included in the meta-analysis and single-institution series included both primary and revision resections as well as noncraniopharyngioma suprasellar pathologies. ${ }^{15,17}$ There remains a lack of consensus and evidence regarding the benefits of TCM versus EEEA in the setting of primary resection. In the current study, we aimed to evaluate the outcomes of TCM and EEEA operations for primary resection of craniopharyngiomas in adults at our institution.

\section{Methods}

Institutional review board exemption through the Indiana University Office of Research Compliance Human Subjects Division was obtained for the planned retrospective review prior to initiating patient identification or chart review. The Indiana University Department of Pathology and Laboratory Medicine surgical case log was queried for all patients with a histopathological diagnosis of craniopharyngioma identified during the period from 2005 through 2015. Noncraniopharyngioma midline suprasellar histopathologies such as pituicytoma, xanthogranuloma, meningioma, pituitary adenoma, glioma lymphoma, and germinoma were excluded from the search. Craniopharyngioma diagnoses before 2005 were excluded to pursue case-control matching in the groups. Pediatric patients $(<$ 18 years of age) were excluded.

Adults undergoing either TCM or an EEEA for recurrent tumors were excluded to avoid confounding bias in results. Preoperative MRI studies were evaluated to accurately determine anteroposterior, craniocaudal, and transverse dimensions of all craniopharyngiomas. Tumor volume was calculated assuming a roughly spherical tumor configuration where tumor volume in cubic centimeters $\left(\mathrm{cm}^{3}\right)=($ anteroposterior $\times$ craniocaudal $\times$ transverse $) / 2$. Large-volume tumors greater than $16 \mathrm{~cm}^{3}(\sim 2$ SDs larger than the combined mean tumor volume of all included patients) were excluded to create a group of tumors that were most likely approachable via TCM and EEEA corridors. None of the included patients had significant lateral extension of their tumors that would have precluded EEEA in this study. One independent neurosurgeon skilled in both EEEA and TCM approaches reviewed the preoperative images and confirmed that these tumors were amenable to both approaches.

The physical clinic records and electronic medical record were reviewed for all included patients. Patients were divided into 2 groups depending on operative records indicating whether TCM (pterional, orbitozygomatic, bifrontal, or transcallosal) or an EEEA (transsphenoidal/ 
transtuberculum/transplanum) had been performed. Notation was made of postoperative closure technique in the EEEA group. As recurrent and revision procedures were excluded, no individual patient was represented in both groups of this study. Extent of resection (EOR) was based off of available postoperative imaging reports by an independent neuroradiologist; postoperative MRI studies were unavailable for 3 patients who underwent TCM. For these 3 patients, intraoperative evaluations of the operating surgeon were used. Reference to operative descriptions of the necessity to perform subtotal resection was documented. MRI reports and operative reports were also reviewed to determine tumor characteristics, including texture (solid, cystic, or mixed).

Patient preoperative records were analyzed to determine presenting symptoms, including headache, visual disturbance, diabetes insipidus (DI), or hypopituitarism. Postoperative hospital and clinic notes were reviewed for outcome data (hospital length of stay, seizures, wound infection, stroke/hemiparesis, CSF leakage, meningitis, permanent DI, hypopituitarism, hydrocephalus, hemorrhage, recurrence, perioperative mortality within 30 days, need for adjuvant therapy or revision surgery, and follow-up length). Visual outcomes were categorized as improved, stable, or deteriorated based on preoperative and postoperative visual field examinations. Chart review of otorhinolaryngological patient records lacked detailed quality scoring data (sinonasal outcome test [SNOT-22]). The rhinologist, however, evaluated and documented the presence of nasal crusting, loss of sense of smell and taste, and persistent drainage during the postoperative follow-up visits in EEEA patients. As no objective data of sinonasal outcomes were measured, no statistical analysis for this metric was performed.

Statistical analysis between groups was performed to determine if significant differences existed between proportions or means of perioperative and outcome data. SPSS software (version 23, IBM Corp.) was used for all data analysis. Descriptive statistics were used to analyze patient demographics, presenting symptoms, perioperative complications, EOR, need for adjuvant therapy, and tumor characteristics. Continuous variables were described with means, medians, and interquartile ranges as appropriate, while categorical variables were presented as frequencies/ percentages. Univariate comparisons were conducted using Student t-tests, chi-square tests, and Fisher exact tests. For all tests, $p$ values $<0.05$ were considered statistically significant.

\section{Results \\ Patient Demographics}

We identified a total of 33 histopathological diagnoses of craniopharyngioma at our institution between 2005 and 2015. Twelve cases were excluded by the established criteria, leaving 21 patients in the study (12 TCM and 9 EEEA operations) (Fig. 1). Both patients removed for size exclusion had undergone TCM operation as their approach. No significant demographic differences in mean patient age, sex, or presenting symptoms were identified. Visual disturbance represented the most prevalent presenting symptom in both groups, while headache was the second most common preoperative complaint (Table 1). No patient presented with isolated DI, but hypopituitarism was not uncommon.

\section{Operative Techniques}

Of the TCM cases, $75.0 \%$ were via a pterional approach and $8.3 \%$ each were via bifrontal, orbitozygomatic, and transcallosal approaches. Within the EEEA cases, all patients underwent a transsphenoidal/transtuberculum approach with tailored transplanum extension. In $88.9 \%$ of EEEA cases, closure used a combined underlay/overlay technique with a pedicled nasoseptal flap. The one case with a simpler closure technique (fat graft alone) without a pedicled nasoseptal flap did not result in CSF leakage postoperatively. In the first 6 years of our study, TCM was solely performed because of the absence of an anterior skull base-trained otorhinolaryngologist. After the arrival of a fellowship-trained rhinologist at our institution, the proportion of EEEA cases increased dramatically over time (Fig. 2).

\section{Tumor Characteristics}

Craniopharyngioma characteristics are enumerated in their entirety in Table 2 . The preoperative tumor volumes were similar between surgical groups in our study, with no significant differences noted. Similarly, tumor consistency on MRI and intraoperative reports were without significant differences between the groups. Tumors in both groups uniformly exhibited suprasellar and retrochiasmatic extension without significant lateral extension beyond the supraclinoid internal carotid artery. The histological subtype noted on pathology reports were predominantly adamantinomatous in both the TCM and EEEA groups, while the papillary histopathology was uncommon and the mixed subtype only occurred once; no significant differences existed between the groups.

\section{Postoperative Complications}

Overall perioperative risk of any negative outcome was equivalent between groups $(66.7 \%$ TCM vs $77.8 \%$ EEEA, $p=0.66$ ). The complete listing of perioperative complications by group is tabulated in Table 3. Permanent DI represented the most frequent overall complication in both groups and affected approximately half of all patients without significant differences between the groups. Panhypopituitarism represented the second most common perioperative complication in our series, also with similar frequency between the groups. CSF leak only occurred within the EEEA cases with the difference trending toward but failing to reach significance $(0.0 \%$ TCM vs 22.2\% EEEA, $\mathrm{p}=0.17$ ). Recurrence, meningitis, stroke/ hemiparesis, and wound infections were rare complications in our series, without significant differences between the groups. No patients in either group experienced documented perioperative seizures, hydrocephalus, postoperative hemorrhage, or perioperative mortality occurring within 30 days of the operation.

\section{Visual Outcomes}

Visual deterioration occurred only within the TCM 


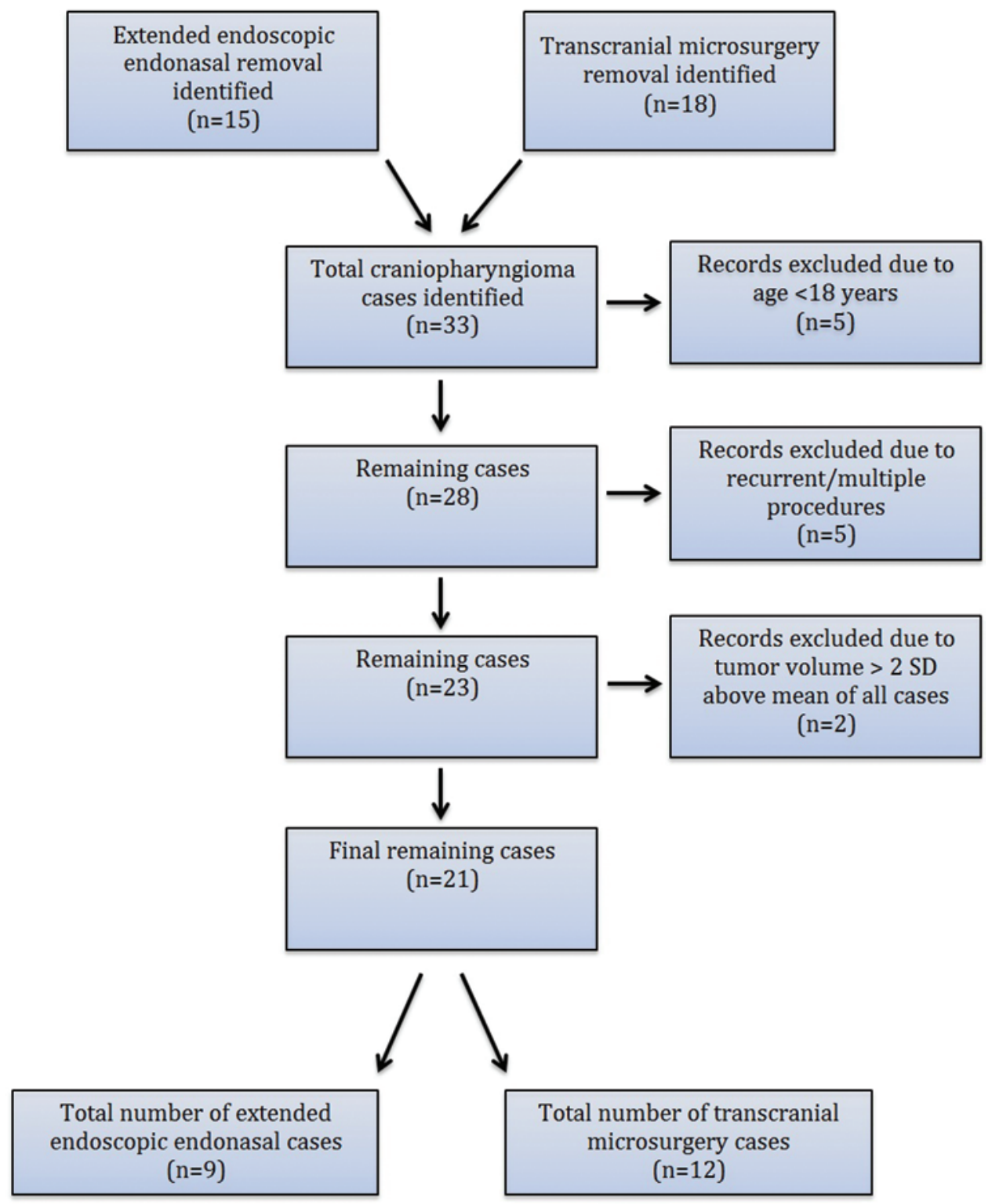

FIG. 1. Flow diagram demonstrating case identification and exclusions to achieve final surgical group numbers.

cases, and the difference did not achieve significance ( $\mathrm{p}$ $=0.23$; Table 4 ). The proportion of patients in each group who either maintained or improved their vision was without any statistical significant difference (75\% TCM vs $100 \%$ EEEA, $p=0.23$ ). While only $25 \%$ of TCM patients experienced improved visual outcomes, $88.9 \%$ of EEEA patients experienced improvement in postoperative vision, and this difference was determined to be statistically significant $(\mathrm{p}=0.0075)$.

\section{Other Outcome Measures}

Additional outcome data are tabulated in their entirety in Table 5. The overall mean length of stay was shorter in the EEEA group than in the TCM group, but this parameter did not achieve significance. More variability in hospital length of stay was noted in the TCM group. Similarly, there was a shorter mean follow-up length in the EEEA group compared with the TCM group, which did not reach significance; again, there was a greater variability within the TCM group. A similar proportion of patients underwent gross-total resection as determined by postoperative imaging and intraoperative inspection; this factor did not reach significance among the 2 groups. Of the patients in whom subtotal resection was performed, a similar proportion went on to require adjuvant therapy in the form of radiotherapy and/or revision surgery without significant differences between these groups.

\section{Discussion}

\section{Patient and Tumor Characteristics}

Our study found no significant differences between TCM and EEEA patients with regard to average age, sex, or types or frequencies of presenting symptoms. Likewise, 
TABLE 1. Demographic data regarding mean patient age, sex, and presenting symptoms

\begin{tabular}{llccc}
\hline \multicolumn{1}{c}{ Variable } & All Cases & TCM & EEEA & p Value \\
\hline No. of cases & 21 & $12(57.1)$ & $9(42.9)$ & \\
\hline Age in yrs & & & & \\
\hline Mean (SD) & $50.1(16.4)$ & $48.3(16.3)$ & $52.4(17.2)$ & 0.59 \\
\hline Median (IQR) & $53(41.5-64.5)$ & $51.5(40.5-62.5)$ & $59(46.8-71.3)$ & 1.00 \\
\hline Male sex & $13(61.9)$ & $7(58.3)$ & $6(66.7)$ & 0.39 \\
\hline Presenting symptom & & & $6(66.7)$ & 1.00 \\
\hline Headache & $11(52.4)$ & $5(41.7)$ & $8(88.9)$ & \\
\hline Visual disturbance & $18(85.7)$ & $10(83.3)$ & $0(0)$ & 1.00 \\
\hline DI & $0(0)$ & $0(0)$ & $3(33.3)$ & \\
\hline Hypopituitarism & $7(33.3)$ & $4(33.3)$ & & \\
\hline
\end{tabular}

IQR = interquartile range

Unless otherwise indicated values are expressed as number of cases (\%).

baseline tumor characteristics including preoperative volume, histopathological subtype, and consistency were all similar without significant differences between the groups. These findings are similar to those reported by Jeswani et al. in their evaluation of outcomes of TCM and EEEA for craniopharyngioma resection. ${ }^{15}$ This homogeneity between surgical groups suggests that our outcomes data reflect differences deriving from the surgical approaches themselves rather than the inherent differences in the type of patient or tumor selected for one approach over the other. It is important to note that all revision/recurrence cases were excluded from our series in an effort to remove any confounding bias derived from increased difficulty in reoperation in a scarred or previously radiated operative field, previously existing injury to the critical structures, or preexisting permanent DI as a previous surgical complication.

\section{Postoperative Endocrine Dysfunction}

While no significant difference was noted in the proportion of our patients whose surgical outcomes were complicated by permanent DI, this did represent a frequent complication, affecting 50\% of TCM patients and $55.5 \%$ of EEEA patients. With regard to permanent DI rates, the rate for TCM patients in our series fared similarly to that of $54.8 \%$ noted in the 2012 meta-analysis by Komotar et al. ${ }^{17}$ and the recent Jeswani et al. series, citing a permanent DI rate of $52.9 \%$ for TCM (no significant difference noted compared with their EEEA group). ${ }^{15}$ Other studies cited rates of postoperative permanent DI following TCM approaches ranging from 23\%, ${ }^{1} 27.2 \%,{ }^{28} 43 \%,{ }^{8}$ to $50 \% .{ }^{34}$ Our $55.5 \%$ postoperative rate of permanent DI in EEEA patients was comparable to the $48.1 \%$ rate cited

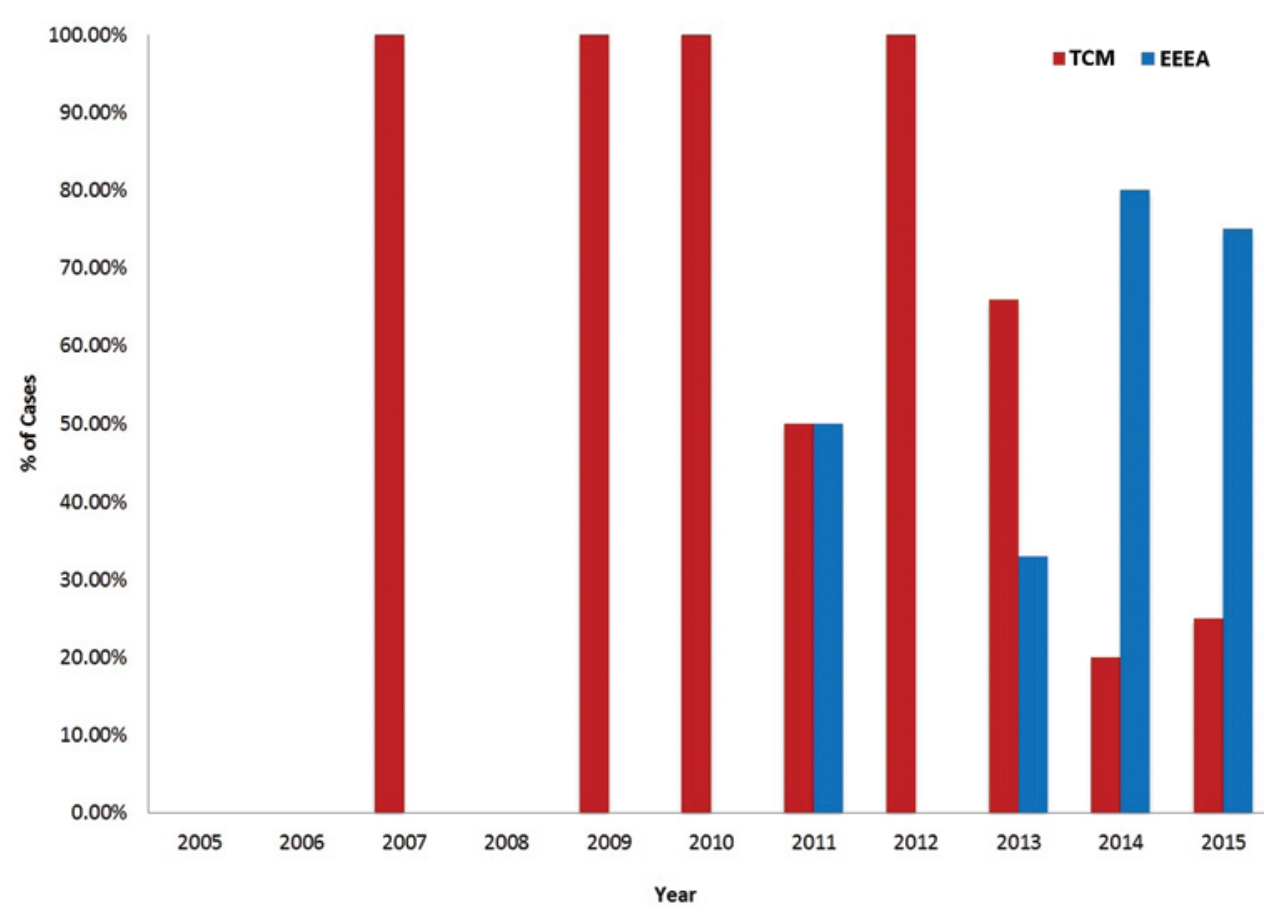

FIG. 2. Bar graph demonstrating percentages of primary craniopharyngioma resections by TCM versus EEEAs at our institution from 2005 to 2015. 
TABLE 2. Tumor size, histopathological subtype, and consistency

\begin{tabular}{lcccc}
\hline \multicolumn{1}{c}{ Variable } & All Cases & TCM & EEEA & p Value \\
\hline No. of cases & 21 & $12(57.1)$ & $9(42.9)$ & \\
\hline Mean tumor vol in $\mathrm{cm}^{3}$ (SD) & $6.5(5.1)$ & $7.8(5.0)$ & $4.6(4.7)$ & 0.16 \\
\hline Pathological type & & & & \\
\hline Adamantinomatous & $17(80.1)$ & $11(91.7)$ & $6(66.6)$ & 0.27 \\
\hline Papillary & $3(14.3)$ & $1(8.3)$ & $2(22.2)$ & 0.55 \\
\hline Mixed & $1(4.8)$ & $0(0)$ & $1(11.1)$ & 0.43 \\
\hline Tumor consistency & & & & \\
\hline Mixed & $14(66.7)$ & $8(66.7)$ & $6(66.6)$ & 1.00 \\
\hline Cystic & $7(33.3)$ & $4(33.3)$ & $3(33.3)$ & 1.00 \\
\hline Solid & $0(0)$ & $0(0)$ & $0(0)$ & 1.00 \\
\hline
\end{tabular}

Unless otherwise indicated values are expressed as number of cases (\%).

by Cavallo et al. in their 2014 series of 103 EEEA patients ${ }^{5}$ and the $44 \%$ rate found in the series of 12 adults by Jane et al. ${ }^{14}$ Our rate of DI in the EEEA group was notably higher, however, than the $27.7 \%$ rate cited in the meta-analysis by the Komotar et al., ${ }^{17}$ the $31.6 \%$ rate found in the Jeswani et al. series,${ }^{15}$ and the $32.3 \%$ rate in the Koutourousiou series of 64 patients. ${ }^{19}$

Panhypopituitarism in our series was less common than permanent DI with rates of $16.7 \%$ in the TCM group and $33.3 \%$ in the EEEA group without the difference achieving statistical significance. Our rates were much lower than those reported in the meta-analysis by Komotar et al. of $48.1 \%$ and $47.1 \%$ for TCM and EEEA, respectively, and their intergroup difference also failed to achieve statistical significance. ${ }^{17}$ Our rates were also lower than those in the Jeswani et al. series of $38.2 \%$ and $42.1 \%$ for TCM and EEEA, respectively. ${ }^{15}$ Other reports of panhypopituitarism following TCM have cited rates similar to those of Komotar et al. and Jeswani et al. ${ }^{1,9,29,33}$ Our EEEA panhypopituitarism rate of $33.3 \%$ was in line with those found by the Koutourousiou series rate of $37.5 \%$ and the Leng et al. series rate of $38 \%,{ }^{20}$ and it was better than the Cavallo et al. rate of $46.2 \% .^{5}$

\section{Visual Outcomes}

We found a statistically significant difference in the rate of postoperative visual improvement between our EEEA and TCM groups ( $88.9 \%$ vs $25 \%$, respectively; $p$ $=0.0075)$. This difference exceeded the significant difference between EEEA and TCM groups reported in the Komotar et al. meta-analysis $(56.2 \%$ vs $33.1 \%$, respectively; $\mathrm{p}<0.003) .{ }^{17}$ Our EEEA findings are similar to those published by Gardner et al. in their 2008 series in which $92 \%$ of patients demonstrated improved vision after EEEA. ${ }^{12}$ High rates of visual improvement following EEEA for craniopharyngiomas have been noted by others as well. ${ }^{5,7,11,26,30}$ The recent single-institution comparison of TCM versus EEEA by Jeswani et al. failed to demonstrate a statistically significant improvement in visual outcomes in their EEEA group. ${ }^{15}$

Visual deterioration occurred only in our TCM group, with $25 \%$ of patients experiencing some degree of visual decline; when compared with that of our EEEA outcomes,
TABLE 3. Postoperative and perioperative complications

\begin{tabular}{lclll}
\hline \multicolumn{1}{c}{ Complication } & $\begin{array}{c}\text { All Cases } \\
(\mathrm{n}=21)\end{array}$ & $\begin{array}{c}\text { TCM } \\
(\mathrm{n}=12)\end{array}$ & $\begin{array}{c}\text { EEEA } \\
(\mathrm{n}=9)\end{array}$ & $\begin{array}{c}\mathrm{p} \\
\text { Value }\end{array}$ \\
\hline Any complication & $15(71.4)$ & $8(66.7)$ & $7(77.8)$ & 0.66 \\
\hline Wound infection & $2(9.5)$ & $1(8.3)$ & $1(11.1)$ & 1.00 \\
\hline Stroke/hemiparesis & $1(4.8)$ & $1(8.3)$ & $0(0)$ & 1.00 \\
\hline CSF leak & $2(9.5)$ & $0(0)$ & $2(22.2)$ & 0.17 \\
\hline Meningitis & $1(4.8)$ & $1(8.3)$ & $0(0)$ & 1.00 \\
\hline Seizures & $0(0)$ & $0(0)$ & $0(0)$ & \\
\hline Permanent DI & $11(52.4)$ & $6(50.0)$ & $5(55.5)$ & 1.00 \\
\hline Panhypopituitarism & $5(23.8)$ & $2(16.7)$ & $3(33.3)$ & 0.61 \\
\hline Hydrocephalus & $0(0)$ & $0(0)$ & $0(0)$ & \\
\hline Hemorrhage & $0(0)$ & $0(0)$ & $0(0)$ & \\
\hline Recurrence & $2(9.5)$ & $1(8.3)$ & $1(11.1)$ & 1.00 \\
\hline Death & $0(0)$ & $0(0)$ & $0(0)$ & \\
\hline Vision deterioration & $3(14.3)$ & $3(25.0)$ & $0(0)$ & 0.23 \\
\hline
\end{tabular}

Unless otherwise indicated values are expressed as number of cases (\%).

this result did not achieve significance $(p=0.23)$. This concern for higher risk of visual deterioration in TCM approaches is supported by the findings of the Komotar et al. meta-analysis where worsening vision was found in $11.3 \%$ of TCM cases versus only in $1.7 \%$ of EEEA cases ( $p<$ 0.003).$^{17}$ Other studies reported similar visual decline rates after TCM of $14.3 \%{ }^{9}$ and $12.8 \% .^{28}$ Our results add to the growing evidence among many institutions that EEEA is superior to TCM approaches with regard to limiting visual deterioration and in maximizing the chance of visual improvement.

The significant improvement in postoperative vision in the EEEA group can be explained by the immediate decompression of the optic apparatus via subchiasmatic removal of the tumor via the transnasal route. However, the transcranial corridor places the optic nerves between the surgeon and the tumor; this phenomenon increases the risk for manipulation of the optic apparatus and resultant visual decline observed in the TCM group.

\section{Cerebrospinal Fluid Leakage}

While there were no CSF leaks observed in our TCM group, our study was likely insufficiently powered to find a statistical difference considering the CSF leak rate of $22.2 \%$ in our EEEA group $(\mathrm{p}=0.17)$. The Komotar et al. meta-analysis found a comparable CSF leakage rate of $18.4 \%$ for EEEA while the rate among the patients who

TABLE 4. Postoperative visual outcomes

\begin{tabular}{lccll}
\hline $\begin{array}{c}\text { Visual } \\
\text { Outcome }\end{array}$ & $\begin{array}{c}\text { All Cases } \\
(\mathrm{n}=21)\end{array}$ & $\begin{array}{c}\text { TCM } \\
(\mathrm{n}=12)\end{array}$ & $\begin{array}{c}\text { EEEA } \\
(\mathrm{n}=9)\end{array}$ & $\begin{array}{c}\mathrm{p} \\
\text { Value }\end{array}$ \\
\hline Improved & $11(52.4)$ & $3(25.0)$ & $8(88.9)$ & $0.0075^{\star}$ \\
\hline Stable & $7(33.3)$ & $6(50.0)$ & $1(11.1)$ & 0.16 \\
\hline Deteriorated & $3(14.3)$ & $3(25.0)$ & $0(0)$ & 0.23 \\
\hline
\end{tabular}

Unless otherwise indicated values are expressed as number of cases (\%). * Statistically significant. 
TABLE 5. Additional outcome measures

\begin{tabular}{llccc}
\hline \multicolumn{1}{c}{ Variable } & All Cases & TCM & EEEA & p Value \\
\hline No. of cases & 21 & $12(57.1)$ & $9(42.9)$ & \\
\hline Mean hospital LOS in days (SD) & $12.6(12.0)$ & $14.4(15.2)$ & $10.1(5.4)$ & 0.38 \\
\hline Mean follow-up time in days (SD) & $313.6(688.1)$ & $375.6(880.2)$ & $216(178.9)$ & 0.57 \\
\hline EOR & & & & 1.00 \\
\hline Gross total & $12(57.1)$ & $7(58.3)$ & $5(55.5)$ & 1.00 \\
\hline$\quad$ Subtotal & $9(42.9)$ & $5(41.7)$ & $4(44.4)$ & 1.00 \\
\hline Therapy required after subtotal resection & $6(66.6)$ & $3(60.0)$ & $3(75.0)$ & 1.00 \\
\hline$\quad$ Revision surgery & $2(33.3)$ & $1(33.3)^{*}$ & $1(33.3)$ & 1.00 \\
\hline$\quad$ Adjuvant radiotherapy & $5(83.3)$ & $3(100)$ & $2(66.6)$ & \\
\hline LOS = length of stay. & & & \\
Unless otherwise indicated values are expressed as number of cases (\%). \\
* One patient in the TCM group required adjuvant radiation that failed and the patient was ultimately treated with revision surgery via an EEEA.
\end{tabular}

underwent TCM was only $2.6 \%$; this difference was significant. ${ }^{17}$ Jeswani et al. also found a significant difference in their EEEA versus TCM CSF leakage rates at 26.3\% and $0 \%$, respectively. ${ }^{15}$ It is not surprising that CSF leakage is more likely to occur after EEEA as this approach opens a high CSF flow corridor between the third ventricle and the nasal cavity.

Our CSF leakage rate after EEEA is comparable to those in other studies evaluating EEEA for craniopharyngioma resection. ${ }^{11,19,30}$ The majority of our patients in the EEEA group underwent a skull base repair performed by an otorhinolaryngologist trained in endoscopic closure utilizing nasoseptal flaps. Institutions with high case volumes of EEEA and routinely utilizing nasoseptal flaps have reported decreasing rates of CSF leakage at $14 \%,{ }^{15} 10.6 \%,{ }^{19}$ and even as low as $4 \%,{ }^{5} 3.8 \%,{ }^{20}$ and $0 \% .{ }^{14}$

\section{Other Complications}

Complications such as wound infection, stroke/hemiparesis, and meningitis were too rare to provide grounds for determining statistical differences between groups, but the latter 2 outcome variables did occur only within the TCM group. None of our patients experienced postoperative hydrocephalus despite it being reported in $15.8 \%$ of EEEA patients by Jeswani et al., ${ }^{15} 12.7 \%$ in EEEA patients by Koutourousiou et al., ${ }^{19}$ and $15.8 \%$ in the Komotar et al. meta-analysis. ${ }^{17}$ Postoperative seizures, hemorrhage, and death within 30 days of surgery also did not occur in our series. For the Komotar et al. meta-analysis, the TCM group had a postoperative seizure rate of $8.5 \%$, hemorrhage rate of $2.0 \%$, and perioperative mortality rate of $3.2 \%$; their EEEA group had a postoperative seizure rate of $0 \%$, hemorrhage rate of $3.2 \%$, and perioperative mortality rate of $1.9 \% .{ }^{17}$ The overall risk of experiencing any complication in our series was not significantly different between groups $(66.7 \%$ TCM vs $77.8 \%$ EEEA; $p=0.66)$. In comparison, Jeswani et al. found a higher risk of experiencing any adverse outcome in the TCM group of $79.4 \%$ and a lower EEEA risk of $52.6 \% .^{15}$

\section{Additional Outcome Data}

There existed no statistically significant difference in the mean hospital length of stay between the surgical groups in our study. It should be noted, however, that less variability was observed in the EEEA group as evidenced by the smaller standard deviation of that group. While this might suggest a more predictable postoperative disposition for EEEA patients, there are numerous confounding variables, including the general preoperative health of the patient, insurance status, time of year of surgery, and availability of family to assist the patient at the time of discharge. Similarly, the mean follow-up time was without significant differences between surgical groups in our study; again, the EEEA group demonstrated much less variability by standard deviation. The same confounders and lack of power would impede a more in-depth analysis of follow-up data as well. Our mean follow-up time was notably shorter than the one published by the Komotar et al. meta-analysis of nearly 2000 days for TCM and 870 days for EEEA. ${ }^{17}$ This discrepancy most likely reflects the statewide neurosurgery referral and surveillance patterns for our institution as well as the relatively short period of time we have had multidisciplinary collaboration with a rhinologist available for EEEA cases (Fig. 2).

EOR was not significantly different between our surgical groups, with roughly the same percentage of cases achieving gross-total resection $(58.3 \%$ TCM vs $55.5 \%$ EEEA, $\mathrm{p}=1.00$ ). It should be noted, however, that a limitation of this present study is a lack of review of our EOR by multiple independent neuroradiologists. Jeswani et al. also failed to demonstrate a significant difference in grosstotal resection rates between TCM and EEEA cases. ${ }^{15}$ While our data do not support a difference in gross-total resection rates between TCM and EEEA, the Komotar et al. meta-analysis did find statistically higher rates in the EEEA group $(66.9 \%$ vs $48.3 \%$, p < 0.003$) .{ }^{17}$ Our TCM rate of gross-total resection was comparable to those reported by other studies. ${ }^{9,29,31-33}$ Likewise, our rate of gross-total resection in EEEA patients was consistent with those reported by other authors, ${ }^{5,7,14,26}$ but some have reported gross-total resection rates as high as $86 \%{ }^{20}$ and $89.6 \%{ }^{12}$ for primary EEEA resections.

Regarding the proportion of our patients who underwent subtotal resection, there were no significant differences found between the TCM and EEEA groups. Furthermore, no significant difference existed in the ultimate 
need for further surgical or radiotherapy in the setting of subtotal resection. Our findings are corroborated by the Komotar et al. meta-analysis and the results of Jeswani et al., which similarly found no significant difference in the need for adjuvant radiotherapy or surgery when comparing TCM to EEEA..$^{15,17}$ Recurrence only occurred in a single patient in each of our surgical groups. The rates of tumor recurrence in the meta-analysis Komotar et al. were 28.2\% in the TCM group and $18.4 \%$ in the EEEA group, and, as in our study, no significant difference was noted. ${ }^{17}$ None of our patients in either group experienced recurrence when gross-total resection had been documented.

\section{Considerations and Limitations}

This present study focused its attention on cases of primary resection of craniopharyngioma in adult patients by TCM and EEEA at our institution in the last decade. In excluding pediatric and revision cases, we traded power in our study for comparing very similar groups of patients who underwent either surgical modality. We felt the reduction in power was a reasonable tradeoff to reduce confounding bias. For example, the EEEA data set in the Komotar et al. meta-analysis was composed of $42.9 \%$ revision cases while their TCM data set included only $18.3 \%$ revision cases, a difference that surprisingly did not achieve significance in their study. ${ }^{17}$ Jeswani et al. admitted to a significant difference between their TCM and EEEA proportion of revision cases (17.6\% vs $42.1 \%$, respectively; $p$ $=0.05$ ); this large difference between groups may have affected their outcome measures..$^{15}$ In the same study, a closer evaluation of the preoperative tumor volume data shows that there was nearly a significant difference in the revision case tumor volumes between groups $\left(4.3 \mathrm{~cm}^{3} \mathrm{TCM}\right.$ vs $10.6 \mathrm{~cm}^{3}$ EEEA; $\left.\mathrm{p}=0.06\right) .{ }^{15}$ By excluding revision cases, our present study avoids these confounders but risked not achieving the power necessary to find likely real outcome differences such as the higher CSF leak rate in EEEA cases and the proportion of postoperative visual deterioration in the TCM group.

As with any retrospective study, this current report is ultimately limited by the quality of data recorded in the clinical medical record. The lack of otorhinolaryngological quality data utilizing the SNOT-22 scoring system limited postoperative sinonasal complaints and hyposmia/ anosmia rate evaluation in our study. However, to the best of our knowledge, none of our patients suffered from disabling forms of these symptoms. There also exists the real risk for selection bias inherent to any surgical retrospective series. In their comparison of TCM and EEEA for craniopharyngioma, Jeswani et al. found that 3 blinded neurosurgeon reviewers were only able to achieve complete concordance in surgical approach selection in $17 \%$ of 53 cases based on preoperative imaging; and they only agreed on the actual approach used in $11 \%$ of the 53 cases..$^{15}$ By chance alone, these reviewers should have agreed $25 \%$ of the time and all selected the actual approach $12.5 \%$ of the time. Jeswani et al. therefore concluded that approach selection was more likely determined by the neurosurgeon's level of expertise and personal preferences rather than because of preoperative imaging or other demographic characteristics. ${ }^{15}$ Ultimately, it would require a random- ized, prospective, dual-armed surgical trial to completely obviate the risk for selection bias in a comparison between TCM and EEEA for primary resection of craniopharyngioma. Because of infrequent disease incidence and ethical considerations, this ideal study is unlikely to occur.

\section{Conclusions}

The prevalence of neurological and otorhinolaryngological surgeons experienced in endoscopic endonasal approaches to the anterior skull base is growing, and this phenomenon has resulted in an increase in the proportion of craniopharyngiomas resected via EEEA at many centers. Most outcome variables between TCM and EEEA are similar. The multidisciplinary EEEA for primary resection of select craniopharyngiomas represents a safe alternative to TCM. We found a significantly higher rate of improved postoperative visual outcomes among EEEA cases when compared with those of TCM where there was a trend toward more visual deterioration. While differences in CSF leakage rates did not achieve statistical difference, CSF leaks were only observed within the EEEA group in our study. CSF leakage may prolong hospitalization or necessitate an additional trip to the operating room for repair, but its negative impact on long-term quality of life is likely to be lower than deterioration in visual outcomes. Large tumors and those with significant lateral extension may still be best managed by means of TCM approaches. While limited by low power, our preliminary results add to the growing evidence that EEEA may be considered the approach of choice for primary craniopharyngioma resection of select tumors in centers with experienced surgeons. Further prospective, multiinstitutional collaboration will be needed to power studies capable of fully evaluating which approach is most appropriate for craniopharyngiomas.

\section{Acknowledgments}

We acknowledge Amy L. Seiler, who assisted in completing chart data acquisition, and Terese M. Stevens, who assisted with IRB submission and compliance.

\section{References}

1. Baskin DS, Wilson CB: Surgical management of craniopharyngiomas. A review of 74 cases. J Neurosurg 65:22-27, 1986

2. Batra PS, Lee J, Barnett SL, Senior BA, Setzen M, Kraus DH: Endoscopic skull base surgery practice patterns: survey of the North American Skull Base Society. Int Forum Allergy Rhinol 3:659-663, 2013

3. Borg A, Kirkman MA, Choi D: Endoscopic endonasal anterior skull base surgery: a systematic review of complications over the past 65 years. World Neurosurg [epub ahead of print], 2016

4. Cappabianca P, Cavallo LM, Colao A, Del Basso De Caro M, Esposito F, Cirillo S, et al: Endoscopic endonasal transsphenoidal approach: outcome analysis of 100 consecutive procedures. Minim Invasive Neurosurg 45:193-200, 2002

5. Cavallo LM, Frank G, Cappabianca P, Solari D, Mazzatenta D, Villa A, et al: The endoscopic endonasal approach for the management of craniopharyngiomas: a series of 103 patients. J Neurosurg 121:100-113, 2014

6. Cavallo LM, Prevedello DM, Solari D, Gardner PA, Esposito 
F, Snyderman CH, et al: Extended endoscopic endonasal transsphenoidal approach for residual or recurrent craniopharyngiomas. J Neurosurg 111:578-589, 2009

7. de Divitiis E, Cavallo LM, Cappabianca P, Esposito F: Extended endoscopic endonasal transsphenoidal approach for the removal of suprasellar tumors: Part 2. Neurosurgery 60:46-59, 2007

8. Elliott RE, Hsieh K, Hochm T, Belitskaya-Levy I, Wisoff J, Wisoff JH: Efficacy and safety of radical resection of primary and recurrent craniopharyngiomas in 86 children. $\mathbf{J}$ Neurosurg Pediatr 5:30-48, 2010

9. Fahlbusch R, Honegger J, Paulus W, Huk W, Buchfelder M: Surgical treatment of craniopharyngiomas: experience with 168 patients. J Neurosurg 90:237-250, 1999

10. Fernandez-Miranda JC, Gardner PA, Snyderman CH, Devaney KO, Strojan P, Suárez C, et al: Craniopharyngioma: a pathologic, clinical, and surgical review. Head Neck 34:1036-1044, 2012

11. Frank G, Pasquini E, Doglietto F, Mazzatenta D, Sciarretta V, Farneti G, et al: The endoscopic extended transsphenoidal approach for craniopharyngiomas. Neurosurgery 59 (1 Suppl 1):ONS75-ONS83, 2006

12. Gardner PA, Kassam AB, Snyderman CH, Carrau RL, Mintz $\mathrm{AH}$, Grahovac S, et al: Outcomes following endoscopic, expanded endonasal resection of suprasellar craniopharyngiomas: a case series. J Neurosurg 109:6-16, 2008

13. Garnett MR, Puget S, Grill J, Sainte-Rose C: Craniopharyngioma. Orphanet J Rare Dis 2:18, 2007

14. Jane JA Jr, Kiehna E, Payne SC, Early SV, Laws ER Jr: Early outcomes of endoscopic transsphenoidal surgery for adult craniopharyngiomas. Neurosurg Focus 28(4):E9, 2010

15. Jeswani S, Nuño M, Wu A, Bonert V, Carmichael JD, Black KL, et al: Comparative analysis of outcomes following craniotomy and expanded endoscopic endonasal transsphenoidal resection of craniopharyngioma and related tumors: a singleinstitution study. J Neurosurg 124:627-638, 2016

16. Komotar RJ, Roguski M, Bruce JN: Surgical management of craniopharyngiomas. J Neurooncol 92:283-296, 2009

17. Komotar RJ, Starke RM, Raper DM, Anand VK, Schwartz TH: Endoscopic endonasal compared with microscopic transsphenoidal and open transcranial resection of craniopharyngiomas. World Neurosurg 77:329-341, 2012

18. Koutourousiou M, Filho FVG, Costacou T, FernandezMiranda JC, Wang EW, Snyderman CH, et al: Pontine encephalocele and abnormalities of the posterior fossa following transclival endoscopic endonasal surgery. J Neurosurg 121:359-366, 2014

19. Koutourousiou M, Gardner PA, Fernandez-Miranda JC, Tyler-Kabara EC, Wang EW, Snyderman CH: Endoscopic endonasal surgery for craniopharyngiomas: surgical outcome in 64 patients. J Neurosurg 119:1194-1207, 2013

20. Leng LZ, Greenfield JP, Souweidane MM, Anand VK, Schwartz TH: Endoscopic, endonasal resection of craniopharyngiomas: analysis of outcome including extent of resection, cerebrospinal fluid leak, return to preoperative productivity, and body mass index. Neurosurgery 70:110-124, 2012

21. Liu JK, Cole CD, Kestle JR, Brockmeyer DL, Walker ML: Cranial base strategies for resection of craniopharyngioma in children. Neurosurg Focus 18(6A):E9, 2005

22. Maira G, Anile C, Rossi GF, Colosimo C: Surgical treatment of craniopharyngiomas: an evaluation of the transsphenoidal and pterional approaches. Neurosurgery 36:715-724, 1995

23. Mamelak AN, Carmichael J, Bonert VH, Cooper O, Melmed $\mathrm{S}$ : Single-surgeon fully endoscopic endonasal transsphenoi- dal surgery: outcomes in three-hundred consecutive cases.

Pituitary 16:393-401, 2013

24. Prasad ML, Perez-Ordonez B: Non-squamous lesions of the nasal cavity, paranasal sinuses and nasopharynx, in Gnepp DR (ed): Diagnostic Surgical Pathology of the Head and Neck, ed 2. Philadelphia: Saunders Elsevier, 2009, p 170

25. Regauer S: Nasopharynx and Waldeyer's ring, in Cardesa A, Slootweg PJ (eds): Pathology of the Head \& Neck. New York: Springer, 2006, p 174

26. Sankhla SK, Jayashankar N, Khan GM: Extended endoscopic endonasal transsphenoidal approach for retrochiasmatic craniopharyngioma: Surgical technique and results. J Pediatr Neurosci 10:308-316, 2015

27. Schwartz TH, Fraser JF, Brown S, Tabaee A, Kacker A, Anand VK: Endoscopic cranial base surgery: classification of operative approaches. Neurosurgery 62:991-1005, 2008

28. Shi XE, Wu B, Fan T, Zhou ZQ, Zhang YL: Craniopharyngioma: surgical experience of 309 cases in China. Clin Neurol Neurosurg 110:151-159, 2008

29. Shirane R, Ching-Chan S, Kusaka Y, Jokura H, Yoshimoto T: Surgical outcomes in 31 patients with craniopharyngiomas extending outside the suprasellar cistern: an evaluation of the frontobasal interhemispheric approach. J Neurosurg 96:704-712, 2002

30. Stamm AC, Vellutini E, Harvey RJ, Nogeira JF Jr, Herman DR: Endoscopic transnasal craniotomy and the resection of craniopharyngioma. Laryngoscope 118:1142-1148, 2008

31. Van Effenterre R, Boch AL: Craniopharyngioma in adults and children: a study of 122 surgical cases. J Neurosurg 97:3-11, 2002

32. Yang I, Sughrue ME, Rutkowski MJ, Kaur R, Ivan ME, Aranda D, et al: Craniopharyngioma: a comparison of tumor control with various treatment strategies. Neurosurg Focus 28(4):E5, 2010

33. Yaşargil MG, Curcic M, Kis M, Siegenthaler G, Teddy PJ, Roth P: Total removal of craniopharyngiomas. Approaches and long-term results in 144 patients. J Neurosurg 73:3-11, 1990

34. Zuccaro G: Radical resection of craniopharyngioma. Childs Nerv Syst 21:679-690, 2005

\section{Disclosures}

The authors report no conflict of interest concerning the materials or methods used in this study or the findings specified in this paper.

\section{Author Contributions}

Conception and design: Cohen-Gadol, Wannemuehler. Acquisition of data: Wannemuehler, Rubel, Hendricks. Analysis and interpretation of data: Wannemuehler, Rubel, Hendricks. Drafting the article: Wannemuehler, Rubel, Hendricks. Critically revising the article: Cohen-Gadol, Wannemuehler, Ting, Payner, Shah. Reviewed submitted version of manuscript: all authors. Approved the final version of the manuscript on behalf of all authors: Cohen-Gadol. Statistical analysis: Wannemuehler, Hendricks. Administrative/technical/material support: Cohen-Gadol. Study supervision: Cohen-Gadol.

\section{Correspondence}

Aaron A. Cohen-Gadol, Department of Neurological Surgery, Goodman Campbell Brain \& Spine, 355 West 16th St., Ste. 5100, Indianapolis, IN 46202. email: acohenmd@gmail.com. 